Europhysics News publishes annually three Meetings Issues: in March, June and November. The June issue contains also a directory section on the composition of the European Physical Society. In the March issue the information received to date is given in full; in the two subsequent issues some entries may be abbreviated and marked by an asterisk ( $\left.{ }^{*}\right)$ indicating that full information can be found in the preceding Meetings Issue of Europhysics News.

The present issue contains details of future events about which notification was received at the EPS Secretariat in Geneva by October 1979. They are listed in date order in two categories - Conferences and Schools. Europhysics Conferences, i.e. meetings organized by EPS, its Divisions and Sections are printed in bold type and boxed; events sponsored by EPS are printed in bold type ; Europhysics Study Conferences are marked by an $\Theta$ preceding the date.
For inclusion in the March 1980 issue organizers should send available details to the EPS Secretariat by 11 February.

\section{Key to Abbreviations}

\section{A : Application deadline}

$\mathrm{Ab}$ : Abstracts deadline

inv: attendance restricted to invited applicants

NP : no proceedings will be published

$P$ : Papers deadline (information on proceedings has not been received)

PP : proceedings will be published

The information is given in the following order:

Date

Venue

Title

A : .../Ab : .../PP, NP or P : Papers deadline / Number of participants inv. / languages other than English Sponsorship / Conference fee.

4 after the date indicates that there has been an important amendment to a previously published entry.

\section{Quantum Electronics Div.}

The International Quantum Electronics Conference to be held in Boston, Mass., 23-26 June, 1980 is organized in conjunction with the EPS Quantum Electronics Division, the Board of which is acting as European programme committee. The Conference will cover the following topics :

New lasers and laser schemes

Non-linear processes and related effects Laser spectroscopy and applications

Picosecond technology, spectroscopy and applications

Coherent optical transient phenomena and applications

Laser chemistry and isotope separation

Laser plasmas and laser fusion

Laser and optical materials

Integrated optics, guided wave devices Quantum optics

ther quantum electronic devices

European contributors are asked to send two copies of their summaries (500 words) and, on separate sheets, their abstracts (35 words) to :

Prof. S. D. Smith, Physics Dept.,

Heriot-Watt University, Riccarton, Currie, Edinburgh EH14 4AS, UK, bv 19 December, 1979.

\section{UNIVERSITY OF OXFORD}

\section{Research Officerships in Nuclear Physics}

The University proposes to appoint two Research Officers in the Department of Nuclear Physics, in the field of experimental nuclear structure physics, for a period of three years, from 1 April 1980 or earlier if possible. Salary according to age on the University Lecturer scale (under review) £ 4,333 to £ 9,581 .

Further particulars may be obtained from the department. Applications (one typed copy) including a statement of qualifications, publications and the names of two referees, should reach :

Professor K. W. Allen,

Department of Nuclear Physics, Keble Road, Oxford OX1 3RH

by 30 November, 1979.

\section{Subscriptions to Europhysics News}

Despite increases in costs, the subscription price to Europhysics News for a complete volume of seven Editorial Issues and three Meetings Issues will remain unchanged in 1980 at $75 \mathrm{Sw} . \mathrm{Fr}$. for institutions and libraries.

The Meetings Issues of Europhysics News have gained a wide reputation for the comprehensive nature of their coverage and the accuracy and topicality of the information that is included. Does your library take advantage of this economic service? For multiple orders, special rates can be arranged by writing to the EPS Secretariat stating number required.

\section{SCUOLA NORMALE SUPERIORE \\ Pisa, Italy}

Post-doctoral position in Theoretical Physics is offered for a period of 10 months from 1 October 1980, for research in one of the fields:

astrophysics, plasma physics, particle physics, and field theory.

Applicants may be of any nationality but must not be over the age of 35 on 15 January, 1980.

The total salary attached to this position is 7 million Italian lire. Successful candidates may apply for a partial reimbursement of travel expenses.

Applications should be sent by 15 January, 1980 to the:

Director of the Scuola Normale Superiore,

Piazza dei Cavalieri, I-56100 Pisa, Italy,

enclosing a curriculum vitae, a detailed programme of study, and letters from three referees. 


\section{Conferences}

\section{0}

2-4 Jan.

Coventry, UK

- 17th Annual Solid State Physics Conference

2-5 Jan.

Hull, UK

Annual Meeting of Association for Science Education Association for Science Education, College Lane, Hatfield, Herts. AL 10 9AA

\section{Jan.-29 Feb.}

Santa Barbara, CA, USA

Gordon Research Conferences

A.M. Cruickshank, Director, Gordon Research Conferences, Pastore Chemical Laboratory, Univ. of Rhode Island, Kingston, RI 02881 100 / inv. / US \$215. -

8-9 Jan. Manchester, UK - Diatomic Metals and Metallic Clusters

\section{4-19 Jan.}

Hirschegg, Austria

Int. Workshop VIII: Gross Properties of Nuclei and Nuclear Excitations

H. Feldmeier, Institut f. Kernphysik, Technische Hochschule, Schlossgartenstr. 9.

D-61 Darmstadt, FRG

A : $31.10 .79 / \mathrm{PP} / 50$

21-24 Jan.

* APS-AAPT Meeting

Chicago, IL, USA

21-25 Jan. Garmisch-Partenkirchen, FRG Liquid Crystals of One- and Two-Dimensional Order and their Applications

W. Helfrich, Inst. f. theoretische Physik, Freie Universität, Arnimallee 3, D-1 Berlin 33

22-25 Jan.

Topical Meeting on Heavy-lon Physics

International Centre for Theoretical Physics, POB 586, I-34100 Trieste

A: 15.12 .79 / US $\$ 50$.

28-30 Jan.

Cambridge, MA, USA

Meeting on X-Ray Astronomy (HEAO-B)

R. Giacconi, Harvard-Smithsonian Center for Astrophysics, 60 Garden Street. Cambridge, MA 02138

$\mathrm{Ab}: 16.11 .79$

28-30 Jan.

Lake Tahoe, NV, USA

5 th Topical Meeting on Integrated and Guided Wave Optics

29-31 Jan.

Estes Park, CO, USA

7 th Conf.: Physics of Compound Semiconductor Interfaces

W. Wilmsen, Dept. of Elect. Engng., Colorado State Univ., Fort Collins, CO 80523

30 Jan.-2 Feb.

Canberra, Australia

12th Australian Meeting of Crystallographers

G.M. McLaughlin, Research School of Chemistry, Australian National University, POB 4. Canberra, A.C.T., 2600

5-6 Feb.

Los Angeles, CA, USA

- Cleaning, Finishing and Coating Processes Seminar

11-14 Feb.

Tvärminne, Finland

Nonlinear and Mechanical Effects of Laser Radiation

S. Stenholm, Dept. of Physics, Univ. of Helsinki NP / 40

13-15 Feb.

San Francisco, CA, USA

* Int. Solid State Circuits Conference

26-28 Feb.

San Diego, CA, USA

-3rd Conf. on Laser and Electro-Optical Systems 2nd Meeting on Inertial Confinement Fusion

26-29 Feb.

London, UK

* Int. Conf. on Submarine Telecomm. Systems

27-29 Feb.

Dortmund, FRG

Teilchenphysik (DPG)

D. Wegener, Lehrstuhl f. Experimentalphysik V der Universităt, Otto-Hahn-Str. D-4600 Dortmund 50

$\mathrm{Ab}: 4.1 .80$

\section{7-29 Feb.}

Physics of the Jovian Magnetosphere

T. W. Hill, Dept. of Space Physics and Astronomy, Rice University, Houston, TX 77001

\section{7-29 Feb.}

Wire Chamber Conference

M. Regler, Institut für Hochenergiephysik, Nikolsdorferg. 18, A-1050 Wien

Vienna, Austria

12-14 March

Versailles, France *Int. Symposium on Distributed Data Bases

12-14 March Arlington, TX, USA 3rd Int. Conf.: Thermoelectric Energy Conversion K.R. Rao, University of Texas, Arlington, TX 76019 Sch. 400.- (students 200.-

3-5 March Austin, TX, USA

8th Austin Symposium on Molecular Structure

E. Boggs, Dept. of Chemistry, Univ. of Texas, Austin, TX 78712

16-18 March

Washington, DC, USA

Particle Accelerator Conference

L. Costrell, Nat. Bureau of Standards, C 333 Radiation Physics, Washington, DC 20234

3-7 March 4 Pittsburgh, PA, USA Analytical Chemistry and Applied Spectroscopy E.S. Hodge. 4400 Fifth Ave., Pittsburgh, PA 15213

\section{3-7 March}

Bielefeld, FRG

44. Physikertagung 1980 (DPG)

- Atomphysik

J.P. Toennies, MPI für Strömungsforschung. Böttingerstr. 4-8, D - 3400 Göttingen

Dünne Schichten

H. Oechsner. Physikalisches Institut der TU Leibnizstr. 4, D 3392 Clausthal-Zellefeld

- Kurzzeitphysik

M. Hugenschmidt, Deutsch-Französisches Forschungsinstitut, Postfach 1560, D-7858 Weil/Rhein

- Massenspektrometrie

$\mathrm{H}$. Hotop, Institut für Experimentalphysik der Universität, Olshausenstr. 40-60, D-2300 Kiel

- Molekülphysik

K. Möbius, FB Physik der Freien Universităt (WE 2), Institut f. Molekülphysik, Boltzmannstr. 20, D-1000 Berlin 33

- Oberflächenphysik

K. Müller, Lehrstuhl für Festkörperphysik, Universität Erlangen-Nürnberg, Erwin-Rommel-Str. 1. D-8520 Erlangen

- Plasma- und Gasentladungsphysik

S. Witkowski, Max-Planck-Gesellschaft, Projektgruppe für Laserforschung. Institutsgelände, D-8046 Garching

- Quantenoptik

A. Laubereau, Lehrstuhl für Experimentalphysik III der Universität, Münzgasse 9, D-8580 Bayreuth

Ab: 1.12 .79

4-6 March

Zutions

P.E. Leuthold, Institut f. Hochfrequenztechnik, ETH, Sternwartstr. 7, Zurich

\section{5-7 March}

Mainz, FRG

Extraterrestrische Physik (DPG)

H.J. Fahr, Institut f. Astrophysik u. Extraterrestrische Forschung der Universität, Auf dem Hügel 71 D-5300 Bonn

Ab: 10.1 .80

5-11 March

Stanford, CA, USA

2nd Novosibirsk/SLAC Conf. on Storage Ring Instrumentation

P. Oddone, Storage Ring. Conf., Bin 12, SLAC, POB 4349, Stanford, CA 94305

9-13 March

Knoxville, TN, USA

2nd Int. Symposium: Gaseous Dielectrics

D. Bouldin, Oak Ridge Nat. Lab. 4500S, H-162, POB X, Oak Ridge, TN 37830

9-14 March

Jerusalem, Israel

* 5 th Int. Congress: International Radiation Protection Association

\section{9-21 March}

15 th Rencontre de Moriond:

Les Arcs, France

- High Energy Hadronic Interactions (9-15.3)

Electroweak Interactions and Unified Theory Predictions (15-21.3)

Tran Thanh Van, LPTPE, Building 211, University Paris XI, F - 91405 Orsay Cedex. PP / $80 /$ inv.

\section{0-12 March}

27 th Int. Scientific Congress on Electronics

20 th Int. Scientific Meeting on Space

Rassegna Internazionale Elettronica Nucleare ed Aerospaziale Via Crescenzio 9, 1-00193 Rome Ab: 15.11.79 / PP / Lit. 50000.

\section{0-13 March}

Munich, FRG

*7. Gemeinschaftstagung der Deutschen Arbeits gemeinschaft für Akustik (DAGA 80)

$\mathrm{Ab}: 1.12 .79$

10-15 March

Palm Coast, FL, USA

Int. Symposium an Atomic, Molecular and Solid State Theory, Collision Phenomena and Computational Quantum Chemistry

Acting Director, Sanibel Symposia, Quantum Theory Project, 365 Williamson Hall,

Univ. of Florida, Gainesville. FL 32611

A: 31.12.79/Ab: $1.2 .80 / P: 20.3 .80 / 150$

17-20 March Palm Coast, FL, USA

Int. Symposium on Fundamentals of Quantum Statistics and Quantum Theory, particularly Density Matrix Formalism

Acting Director, Sanibel Symposia, Quantum Theory Project, 365 Williamson Hall,

Univ. of Florida, Gainesville, FL 32611

A: $31.12 .79 / A b: 1.2 .80 / P P: 20.3 .80 / 100$

17-21 March

Alabama Gulf Coast, USA

Meeting of American Crystallographic Association

Ms. E.E. Snider. American Crystallographic Assoc., 335 E. 45th Str. New York, NY 10017

17-21 March

Vienna, Austria

-6th Int. Congress: Data Processing in Europe

17-22 March

Munich, FRG

P.v. Brentano, Institut f. Kernphysik der Universität Zülpicherstr. 77, D-5000 Köln 41

$\mathrm{Ab}: 14.12 .79$

17-24 March

Europhysics Study Conf.: Unification of the Fundamental Interactions

S. Ferrara, J. Ellis, P. Nieuwenhuizen, ENS, Lab. de Physique Théorique, 24, rue Lhomond.

F-75231, Paris Cedex 05

18-21 March

Toulouse, France

Journées Industrielles de la Société Française de Physique

J. Coulom, CERT, B.P. 4025

F-31055 Toulouse Cedex

A: 18.3.80 / Ab: 31.1 .80 / PP: 21.3.80 / Engl., French / FF 650.- (IOM \& mbrs. SFP)

FF 850. - (others)

23-26 March

Brussels, Belgium

European Conf on Innovation in Primary Publication: Impact on Producers and Users (EURIM 4)

ASLIB, 3 Belgrave Square, London SW1X 8PL, GB

24-25 March

London, UK

Radio Transmitters and Modulation Techniques IEE Conf. Dept., Savoy Place, London WC2R OBL

\section{4-26 March}

Microstructure of Carbon and Graphite

Glasgow, UK

T. Baird, Dept. of Chemistry, University of Glasgow, Glasgow G12 800

Ab: $1.11 .79 / \mathrm{PP}$

\section{4-26 March}

Physik der Hochpolymeren

Leoben, Austria

R. Bonart, FB Physik der Universität, Universitätsstrasse 31, D 8400 Regensburg

Ab: 1.12 .79

\section{4-27 March 4}

Orlando, FL, USA

2nd Int. Conf. on Magnetic Fluids

M. Zahn, Dept. of Electrical Engineering, University of Florida, Gainesville, FL 32611

Ab: 30.5 .79 / PP: $14.9 .79 / 80$

24-28 March

Freudenstadt, FRG

DPG Tagung

- Halbleiterphysik

J. Treusch, Institut für Physik der Universität, Postfach 500500, D - 4600 Dortmund 50

- Magnetismus (Grundlagen)

E. Müller-Hartmann, Institut für Theoretische Physik der Universität, Zülpicher Str. 77. 
12th National Atomic and Molecular Physics Conf. and Workshop

A. Corney, The Clarendon Laboratory, Parks Road, Oxford

$A b: 21.1 .80 / P P / 100$

24-29 March

Ronda (Malaga), Spain

8 th Int. Winter Meeting on Fundamental Physics

A. Ferrando, Altas Energias, Junta Energia Nuclear. Madrid 3

A : $15.2 .80 / P P / 70$

25-28 March

Durham, UK

25-28 March
-Data Bases in Distributed Systems (incl. Social Impacts) hFl. 900.-

26-28 March

- Phase Transitions in Molecular Solids

Exeter, UK

26-28 March

Giessen, FRG

Physikdidaktik (DPG)

W. Kuhn, Institut f. Didaktik der Physik, Universität Giessen, Karl-Glöckner-Str. 21, D-6300 Giessen $A b: 1.12 .79$

26-29 March

Paris, France

Symposium on Archaeometry

Symposium Secretary, C.R.G. Garchy,

F-58150 Pouilly-sur-Loire

27-28 March

Resonance Studies of Conductors and Semiconductors

J.E. Cousins, Dept. of Physics, Univ. of Exeter, Exeter EX4 4OL

A: $27.2 .80 / \mathrm{Ab}: 27.2 .80 / \mathrm{NP} / 75 / \mathrm{f} 10$. -

31 March-2 April

Madrid, Spain

3rd Int. Conf. on Physico-Chemical Hydrodynamics

Manuel G. Velarde, Facultad de Ciencias C-3, Universidad Autónoma de Madrid,

Cantoblanco (Madrid)

A : Dec. $79 / 200 /$ US \$ 50 .

31 March - 2 April

Brighton, UK

4th Int. Conf. and Exhibition: Computers in Design and Engineering (CAD 80)

IPC Science and Technology Press Ltd., POB 63. Westbury House, Bury Street

Guildford, Surrey GU2 5BH

PP : 21.12 .79

April
Annual Scanning Electron Microscopy Symposium

April
Annual Scanning Electron Microscopy Symposium Om Johari. POB 66507. AMF O'Hare, IL 60666

1-3 April St. Andrews, UK * Spring Meeting: Crystallography Group, IoP

1-4 April

Toulouse, France

*6th Int. Conf. on Solid State Dosimetry

7-11 April

Washington, DC, USA

Int. Conf. on Optical Computing

S. Horvitz, POB 274, Waterford, CT 06385

7-11 April

Nagoya, Japan

* 4 th Kiev Int. Conf. on Plasma Theory

4 th Int. Congress on Waves and Instabilities in Plasmas

8-12 April

Vienna, Austria

1 st World Congress on Biomaterials

K. Copeland, University College, Gower Street, London WC 1

9-11 April

Denver, CO, USA

Int. Conf. on Acoustics, Speech and Signal Processing

J.R. Ashley. Dept. of Electr. and Computer Engineering, University of Colorado 1100 14th Street, Denver, CO 80202

9-11 April

Durham, UK

* Modern Spectroscopic Techniques for the Investigation of Solids

\section{9-11 April 4 \\ Antwerp, Belgium \\ Annual Meeting: Condensed Matter} Division of EPS

J. Devreese, RUCA, Groenenborger-

Iaan 171, B-2020 Antwerp

Ab: 15.1.80/ PP / bFr. 3000.-

Proformat for participants in the:

\section{European Physical Society 1980 Annual Meeting of the Condensed Matter Division * 9-11 April 1980, Antwerp, Belgium}

Name

\section{Affiliation}

\section{Address}

I expect to attend the meeting

I expect to present a contributed paper ${ }^{\star \star}$ on the following topic:

I want more information, please put my name on the mailing list

Return copy to Prof. Dr. J.T. Devreese, Chairman of the Conference,

Universiteit Antwerpen (R.U.C.A.), Groenenborgerlaan 171,

B-2020 ANTWERPEN (Belgium).

The conference will deal with all aspects of the physics of condensed matter There will be a series of symposia on the most important recent developments in the physics of condensed matter. The conference will be open to all physicists. (For details on topics, see announcement in the August issue of Europhysics News, p. 12.)

** Deadline for the receipt of abstracts: 15 January, 1980.

9-11 April

Int. Symposium: Physical Properties of Actinides and Related 4f-Materials

B. Reihl, Laboratorium für Festkörperphysik, Hönggerberg, $\mathrm{CH}-8093$ Zürich

A : 10.1.80/Ab: 10.1.80/PP: $10.3 .80 / 150 /$ incl. proc. SFr. 150.- (IOM), Sfr. 180.(others)

\section{1-14 April}

Vienna, Austria

6th Annual European Geophysical Society Meeting 6 th Annual EGS Meeting, c/o Institut $f$. Meteorologie u. Geophysik, Universität Wien. Hohe Warte 38, A-1190 Vienna

14-16 April

Pisa, Italy

European Conf. on the Dynamics of Excited States R. Moccia Istituto di Chimica Fisica dell'Università Via Risorgimento 35, I-56100 Pisa

14-16 April

Int. Conf.: The Rational Use of Energy

A.I.M., Rue Saint-Gilles 31, B-4000 Liège

\section{4-16 April}

Semi-insulating III-V Materials

P.R. Jay, Plessey Research Ltd., Caswell, Towcester. Northants.

$\mathrm{Ab}: 1.11 .79 / \mathrm{PP}$

\section{4-17 Apri}

Low Energy lon Beams - 2 (LEIB - 2)

The Meetings Officer. The Institute of Physics 47 Belgrave Square, London SW $1 \times 80 X$ A: $14.3 .80 / A b: 14.12 .79 / P P: 14.4 .80 / 150$

14-18 April

Taormina, Italy

Wave Propagation in Viscoelastic Media

F. Mainardi, Faculty of Sciences, Istituto di Fisica, Università di Bologna, Via Irnerio 46 -40126 Bologna

Ab: $15.12 .79 / 50$

14-18 April Budap
2nd Int. Conf. on Multiphoton Processes

Budapest, Hungary

$Z$. Horvath, Organizing Committee, Central Res. Inst. for Physics, POB 49, H-1525 Budapest

A: $1.2 .80 / \mathrm{Ab}: 1.2 .80 / \mathrm{PP} / 200 /$ inv. / IUPAP Sfr. 160. - (IOM), 180. - (mbrs 4b), 200.(others)

14-18 April

Bournemouth, UK

5 th ESA Symposium on European Sounding Rocket and Balloon Programmes and Related Research

T. Halvorsen, ESA, 8-10 rue Mario Nikis, F-75738 Paris 15

A: 20.2.80/Ab: End Sept. 79 / PP: $18.4 .80 / 150 /$ E 10 . -

15-17 April

2nd European Symposium on Photovoltaic Generators in Space

H. Lösch, DFVLR/BPT, Postfach 906058 D-5000 Köln 90

A: $30.1 .80 / A b: 1.12 .79 / P P: 17.4 .80 / 150$
15-18 April
*Communications Equipment and Systems

15-18 April

Tsukuba, Japan

Int. Symposium on Physics in Open-Ended Systems

16-17 April

London, UK

High Temperature Plasma Physics and its Applications

The Executive Secretary. The Royal Society, 6 Carlton House Terrace, London SW1Y 5AG

16-18 April

Magnetismus

K. Schüler. Thyssen Edelstahlwerke AG, Ostkirchstr. 177, D-4600 Dortmund 4 $A b: 30.11 .79$

16-18 April

Manchester, UK

Trends in Nuclear Structure Physics

A.R. Barnett, Dept. of Physics, Univ. of Manchester Manchester M13 9PL

A: $3.3 .80 / A b: 18.2 .80 / 200$

16-18 April

Oporto, Portugal

2nd Meeting of Portuguese Physical Society

Secretariado de Fisica-80, Lab, de Fisica,

Faculdade de Sciencias, Univ. do Porto, P-4000 Porto

Ab: 15.2.80/Port., Engl., French / Esc. 500.-

18-20 April Birmingham, UK

Education Group Annual Conf. : Writing about Physics The Meetings Officer, The Institute of Physics, 47 Belgrave Square, London SW $1 \times 80 X$

A: 15.3.80/Ab: Jan. 80

21-24 April

Washington, DC, USA

APS General Meeting

Prican Physical Society, 335 E. 45th Street, New York, NY 10017

\section{1-24 April}

18 th Int. Magnetics Conf. (INTERMAG)

D.I. Gordon, Naval Surface Weapons Center, White Oak Lab., Silver Spring, MD 20910

Ab: 7.12 .79

21-25 April Garmisch-Partenkirchen, FRG

* 4th Int. Conf. on Plasma Surface Interaction on Controlled Fusion Devices

21-25 April Noordwijkerhout, NL

10th Europhysics Conference on Macromolecular Physics - Structure and Motion in Polymeric Glasses

J. Heijboer, Division of Technology for Society, TNO, POB 217, NL-2600 AE Delft

A : $15.1 .80 / A b: 30.10 .79 / P$ : 15.1 .80 $100 / \mathrm{hFI} .560$. - all incl.

21-29 April

3rd Seminar on Noise Reduction

Budapest, Hungary

Mrs. V. Vadasz, Optical, Acoustical and Filmtechnica Society, Ankerköz 1. H-1061 Budapest

- For full details please see June 1979 issue of Europhysics News 
22-24 April

Strasbourg, France

Nuclear Behaviour at High Angular Momentum

B. Haas, CRN-PNIN, rue du Loess 23 . F-67037 Strasbourg Cedex

$120 /$ inv.

22-25 April

Pont-à-Mousson, France

1980 Optical Conf. : Horizons de l'Optique

C. Imbert, Institut d'Optique, B.P. 43. F-91406 Orsay Cedex

PP / $300 /$ Engl., French /FF 300.-

25-30 April Balatonaliga, Hungary
10th Hungarian Diffraction Conference

Mrs. E. Nagy, Roland Eötvös Physical Society, POB 240, $\mathrm{H}-1368$ Budapest

150 / inv. / Sfr. 400 .

\section{8-30 April}

Arlington, VA, USA

Int Radar Conference

R.T. Hill, Conf. Office, 777 14th Str. NW, Suite 917 Washington, DC 20005

28-30 April

Strasbourg, France

Int. Colloquium : Economic Effects of Space and Other Advanced Technologies

G. Niederau, European Space Agency, 8-10 rue Mario Nikis, F-75738 Paris 15 PP

28-30 April Perchtoldsdorf, Vienna, Austria Symposium on Sputtering

W. Husinsky, Symp. on Sputtering, Postf. 69, A-1043 Vienna

A : 1.12.79/ Ab: 1.12.79/PP/150/Sch. 750. -

28 April - 2 May Linz, Austria

IFIP Working Conf.: Firmware, Microprogramming. Restructurable Hardware

H. Hayman, POB 639, Silver Spring MD 20901

30 April-3 May

Sarasota, FL, USA

Topical Meeting on Recent Advances in Vision

Optical Society of America, 1816 Jefferson Place, NW, Washington, DC 20036

Ab: 15.1 .80 / US \$ 50.-

May

Paris, France

2nd IAEA Advisory Group Meeting on Atomic and Molecular Data for Fusion

K. Katsonis, Nuclear Data Section, IAEA, Kärntner Ring 11, POB 590, A-1011 Vienna

1-3 May Nashville, TN, USA Vanderbilt Symposium on $\mathrm{e}^{+} \mathrm{e}^{-}$Interactions at High Energies

R. Panvini, Physics Dept. Vanderbilt University Nashville, TN 37235

5-7 May

Gaithersburg, MD, USA

Basic Optical Properties of Materials

Mrs. K. Stang, Materials Bldg. B-348 National Bureau of Standards, Washington, DC 20234

$A b: 7.1 .80$

6-8 May

Ajaccio, Corsica, France

Conf. sur les Matériaux pour la Conversion Photothermique de I'Energie Solaire

M. Salvi, CENG Grenoble, B.P. 85 ,

F-38041 Grenoble Cedex

$A b: 15.1 .80$ / PP: 1.5.80/ Engl., French

6-8 May

La Baule, France

7 th Int. Symposium on Computer Architecture

J. Lenfant, IRISA, Université de Rennes, F-35042 Rennes Cedex

$A b: 1.8 .79 / P: 1.10 .79$

6-8 May San Diego, CA, USA

25th Nat. SAMPE Symposium and Exhibition

Marge Smith, SAMPE, POB 613, Azusa, CA 91702

Ab: 1.7 .79

6-9 May

Berlin (West)

2nd Int. Conf. on Superconducting Quantum Devices

IC SQUID Secretariat, PTB-Institut Berlin Abbestrasse 2-12, D-1000 Berlin 10

Ab: 31.1.80/PP / $300 /$ DM 220. -

7-9 May Delft, Netherlands
6th Solid-Vacuum Interface Conf.: Application of

Delft, Netherlands Advanced Surface Spectroscopic Techniques

Mrs. Thea van de Laak. Applied Physics Department, Delft University of Technology, Lorentzweg 1. NL-2628 CJ Delft

12-16 May

Dublin, Eire

Working Seminar on Problems in Nonlinear Continuum Mechanics

Director, School of Theor. Physics, Dublin Inst. for Advanced Studies, 10 Burlington Rd., Dublin 4 50 / irf 20.-
13-16 May

Kailua-Kona, HI, USA

The Satellites of Jupiter

D. Morrison, Institute for Astronomy,

2680 Woodlawn Dr., Honolulu, HI 96822

19-22 May

Madison, Wisconsin, USA

Int. Conference on Plasma Science

J.L. Shohet, Dept. of Electrical and Computer Engineering, Univ. of Wisconsin-Madison, 1425 Johnson Drive, Madison, WI 53706

28-30 May 4

Washington, DC, USA

International Microwave Symposium

L.R. Whicker, Naval Research Laboratory,

Code 5250, Washington, DC 20375

June

8 th Int. Liquid Crystal Conference

Kyoto, Japan

G.M. Brown, Liquid Crystal Institute, Kent State University, Kent, OH 44242, USA

2-4 June

Cape Cod, MA, USA

Topical Meeting on Hologram Interferometry and Speckle Metrology

Optical Society of America, Hologram Interferometry Meeting. 1816 Jefferson Place,

N.W., Washington, DC 20036

Ab : 1.2 .80

2-6 June

Spain

XI GIFT Seminar on Theoretical Physics: Electroweak Interactions

J. Sánchez-Guillén, Faculty of Sciences, University of Zaragoza

A: 31.3.80/PP

2-14 June

Budapest, Hungary

23rd COSPAR Plenary Meeting and Seminars

Z. Niemirowicz, COSPAR Secretariat, 51 bd. de Montmorency, F-75016 Paris

Ab: 1.2.80 / PP / 700 / English, French / US \$ 60. -

\section{3-5 June}

Int. Conf.: Networks 80

London, UK

Online, Cleveland Road, Uxbridge UB8 2DD

$\mathrm{Ab}: 28.11 .79 / \mathrm{PP}: 7.3 .80$

3-6 June

8th Int. Cryogenic Engineering Conf. (ICEC 8)

C. Rizzuto, ICEC 8, C.P. 569, I-16100 Genoa

$\mathrm{Ab}: 10.12 .79$

3-6 June

Sesimbra, Portugal

9th European Symposium on Few Body

Problems in Nuclear and Particle Physics

A.C. Fonseca, Secretariado da Conferencia, Av. Gama Pinto 2, P-1699 Lisboa Codex

A : $1.3 .80 /$ PP: $1.3 .80 / 100 /$ Esc. 1000 . (IOM), 1500.- (others)

5-9 June Priština, Yugoslavia

XXIV ETAN Conf. (all branches of acoustics)

P. Pravica, Electrotechnical Faculty, Bulevar Revolucije 73, YU-11000 Belgrade

8-13 June Las Vegas, Nevada, USA Annual National Meeting: American Nuclear Society David G. Pettengill, American Nuclear Society, 555 North Kensington Ave. La Grange Park, IL 60525

8-14 June

Sitges, Spain

Int. Symposium on Systems Far from Equilibrium L. Garrido, Faculty of Physics. University of Barcelona Diagonal 647, Barcelona-28

8-19 June

Trieste, Italy

Meeting on System Theory and Optimization International Centre for Theoretical Physics. POB 586, 1-34100 Trieste

\section{9-12 June}

Lund, Sweden

2nd Int. Conf.: Particle-Induced X-Ray Emission and its Analytical Applications

Org. Comm., PIXE Conf., Dept. of Nuclear Physics, Lund Institute of Technology, Sölvegatan 14. S-22362 Lund

9-12 June 4

Catania, Italy

5th European Tandem Conference

D. Vinciguerra, LNS, Corso Italia 57, I-95129 Catania

9-13 June

Lund, Sweden

Nobel Symposium : Nuclei at Very High Spins

Sven Gösta Nilsson, University of Lund, Box 725. S-220 07 Lund 7

50 / inv.

\section{9-13 June}

Symposium on Topical Questions in QCD

Hoyer, NORDITA, Blegdan
DK-2100 Copenhagen

10-12 June

Turku, Finland

Scandinavian Acoustical Meeting

J. Starck, Institute of Occupational Health, Laajaniityntie 1, SF-01620 Vantaa 62

11-13 June Cleveland, $\mathrm{OH}$, USA

Int. Symposium on the Statistical Mechanics of Phase Transitions in Polymers

Philip L. Taylor, Physics Dept., Case Western Reserve University, Cleveland, $\mathrm{OH} 44106$

$\mathrm{Ab}: 31.12 .79$

11-14 June 4

Bologna, Italy

AU Symposium: The Origin of Cosmic Rays

F. Pacini, Istituto di Astronomia, Largo Enrico Fermi 5 1-50125 Firenze

IAU, IUPAP

16-18 June

Ithaca, NY, USA

Phys. Electronics Conference

T.N. Rhodin, 217 Clark Hall, Cornell University, Ithaca, NY 14853

$\mathrm{Ab}: 25.4 .80$

16-19 June

Hamilton, Ont., Canada

Congress of Canadian Association of Physicists

Mrs. M. Jento, Canadian Association of Physicists,

151 Slater Str., Suite 903.

Ottawa, Ontario, Canada K1P $5 \mathrm{H} 3$

16-20 June Uppsala, Sweden

Conference on Experimentation at LEP

T. Ekelöf, EP Div., CERN, CH-1211 Geneva 23

A: $20.2 .80 / A b: 20.2 .80 / P P$

Cambridge, MA, USA

7 th Int. Conf. on MHD Electrical Power Generation J.F. Louis, MIT 31-254, Cambridge, MA 02139

$\mathrm{Ab}: \mathbf{7 . 1 . 8 0 / P P : 2 2 . 3 . 8 0}$

18-19 June

London, UK

Meeting on NMR Spectroscopy in Solids

Executive Secretary, The Royal Society.

6 Carlton House Terrace, London SW1Y 5AG

18-20 June

Cape Cod, MA, USA

Topical Meeting on Picosecond Phenomena

Optical Society of America, 1816 Jefferson

Place, N.W., Washington, DC 20036

Ab: 8.2 .80

22-27 June

Bruges, Belgium

11 th Int. Symposium on Multiparticle Dynamics

F. Verbeure, Dept. of Physics, U.I.A., B-2610 Wilrijk $\mathrm{PP} / 70 /$ inv

23-25 June

Heat Transfer and Fluid Mechanics

Mrs. S.M. Paul, University of Southern California,

School of Engineering, University Park Los Angeles, CA 90007

23-26 June

Troms $\phi$, Norway

Annual Meeting: Norwegian Physical Society

O. Holt, I.M.R., University of Troms $\varnothing$, POB 953 , N-9001 Troms $\varnothing$

A : 1.5.80 / Ab: 1.5 .80 / NP: 1.5 .80 / Engl., Norw. / nKr. 150 . -

23-26 June

Aboard ship, Aegean Sea

Resonant Behaviour of Heavy lon Systems

Tandem Accelerator Laboratory, NRC Demokritos, Agia Paraskevi-Attikis, Greece

A: 15.2.80/Ab: $15.2 .80 / \mathrm{PP} / 70 /$ US $\$ 100$.

23-26 June

Boston, MA, USA

11 th Int. Quantum Electronics Conference

S.D. Smith, Heriot Watt University, Riccarton, Currie, Edinburgh EH14 4 AS, GB

Ab: 19.12 .79 (see note on front page)

23-27 June

Kailua-Kona, HI, USA

Infrared Astronomy

D.P. Cruikshank, Institute for Astronomy, University of Hawaii at Manoa, 2680 Woodlawn Drive. Honolulu, HI 96822

23-27 June

Braunschweig, FRG

Precision Electromagnetic Measurements (CPEM 80)

H. Capptuller, PTB, Bundesallee 100.

D-3300 Braunschweig 


\section{3-28 June}

Bressanone, Italy

Symposium on Nucleon-Antinucleon Interactions

M. Cresti, Università degli Studi di Padova, Istituto di Fisica Galileo Galilei, Via F. Marzolo 6.

I-35100 Padua

US $\$ 30 .-$ (incl. proc.)

27 June - 1 July

27 June - 1 July Tokyo, Japan Conter Tokyo 100

$\mathrm{Ab}: 15.6 .79 / \mathrm{P}: 15.1 .80$

30 June - 4 July Conf. on Metallic Glasses: Science and Technology K. Tompa, POB 49, $\mathrm{H}-1525$ Budapest 114

A: $30.4 .80 / \mathrm{Ab}: 31.3 .80 / \mathrm{PP}: 30.6 .80 / 250$ US $\$ 100$.

30 June - 4 July

Antwerp, Belgium

7th European Thermophysical Properties Conf.

R. de Coninck, Laboratoria van het SCK/CEN, Boeretang 200, B-2400 Mol

A : $1.5 .80 / \mathrm{Ab}: \mathbf{1 5} .12 .79 / \mathrm{PP} / 200$ bFr. 3000-3500

30 June-4 July

7 th Trieste Conference on Particle Physics

International Centre for Theoretical Physics. POB 586, I-34100 Trieste

July Toronto, Ont., Canada

6 th Int. Colloquium on UV and X-Ray Spectroscopy of Astrophysical and Laboratory Plasmas

R.W. Nicholls, Center for Research in Experimental Space Science, York Univ., 4700 Keele Street, Downsview, Ont., Canada M3J 1P3 50 / IAU

1-10 July

Brussels, Belgium

8th IAEA Int. Conf. on Plasma Physics and Controlled Nuclear Fusion Research

R. Najar, Conf. Service Section, IAEA, POB 100 A-1400 Vienna

Ab: 4.2 .80

7-9 July

Radio Spectrum Conservation Techniques

$\mathrm{Ab}: 30.7 .79 / \mathrm{P}: 4.1 .80$

Grenoble, France
4th Int. Conf. on Liquid and Amorphous Metals

G-11 July
4th Int. Conf. on Liquid and Amorphous Mrance

LAM 4, Conf. Secretary, ENSEEG, B.P. 44, F-38401 Saint-Martin-d'Heres

A : 1.5.80/Ab: 1.1.80/PP: 15.5.80/500/Engl., French / FF 700.-

7-11 July

Berlin (West)

5 th Int. Conf. on Spectral Line Shapes (5th ICSLS)

N. Nubbemeyer, 5th ICSLS, PTB, Institut Berlin, Abbestrasse 2-12, D-1000 Berlin 10

Ab $: 1.5 .80$ / IUPAP / DM 150.-

7-11 July CERN, Geneva, Switzerland

XI Int. Conf. on High Energy Accelerators

Miss D.A. Caton, Scientific Conference Secretariat, CERN, CH-1211 Geneva 23

$A b: 14.3 .80 / 350 /$ inv.

7-17 July Paris, France

es the Upper Mantle:

Their Role in Geodynamic Processes

Special Symposium:

Bilan général du programme international de géodynamique et prospectives

Microplate Tectonics

P. Melchior, Secretary General HUGG, Observatoire IUGG Royal, Av. Circulaire 3, B-1180 Brussels

9-23 July

Chania, Crete, Greece

Workshop on Nonlinear Evolution Equations and Dynamical Systems

A. Verganelakis, Nuclear Research Center "Demokritos", Aghia Paraskevi, Attiki, Athens

Copy to: F. Calogero, Dept. of Appl. Mathematics, Queen Mary College, Mile End Road, London E1 4NS

A: 28.2.80/70/US \$ 450.- all incl.

14-16 July Snowmass, CO, USA

13th Fluid and Plasma Dynamics Conf.

15th Thermophysics Conference

Meetings Dept., American Inst. of Aeronautics and Astronautics, 1290 Ave. of the Americas, New York, NY 10019

14-19 July Jena, DDR

9th Int. Conf. on General Relativity and Gravity

E. Schmutzer, GR 9. Sektion Physik, Friedrich-SchillerUniversität, Max-Wien-Platz 1, DDR-69 Jena
15-19 July

Clermont Ferrand, France

International Cloud Physics Conference

Melchior, Secretary General IUGG, Observatoire Royal, Av. Circulaire, 3, B-1180 Brussels

IUGG, IAMAP

17-23 July

Madison, WI, USA

20th Int Conf. on High Energy Physics

Conf. Secr., High Energy Physics Conf., University of Wisconsin, 1150 University Ave.

Madison, W153706

A: $15.1 .80 /$ inv.

20-26 July

Ithaca, NY, USA

Cornell Symposium on Liquid and Solid ${ }^{3} \mathrm{He}$

Robert C. Richardson, Lab of Atomic and Solid State

Physics, Cornell University, Ithaca, NY 14853

21-25 July

Berlin (West)

5 th Int. Conference on Hyperfine Interactions

G. Kaindl, Sonderforschungsbereich 161, Hyperfeinwechselwirkungen, Freie Universităt Berlin, Hüninger Strasse 44, D-1 Berlin 33

22-25 July

Kyoto, Japan

AU Symposium: Fundamental Problems in the Theory of Stellar Evolution

D. Sugimoto, Dept. of Earth Science and Astronomy, Univ. of Tokyo, 3-8-1. Komaba, Meguro-Ku. Tokyo 153

PP / IAU

\section{7-31 July}

Minneapolis, MN, USA

2nd Annual Meeting: American Association of Physicists in Medicine

Executive Director, American Assoc. of Physicists in Medicine, $111 \mathrm{E}$. Wacker Drive,

Chicago, IL 60601, USA

28 July - 1 Aug.

Liège, Belgium

5 th European Regional Astronomy Meeting: Variability in Stars and Galaxies

P. Ledoux, Institut d'Astrophysique, Université de AL Liège, Ave. de Cointe 5, B-4200 Ogrée

28 July - 1 Aug.

6 th European Crystallographic Meeting

ECM-6, Ultramar Express, S.A., Gran Via, 591, 4. Barcelona 7

August

Los Alamos, NM, USA

IAU Colloquium No. 58: Stellar Hydrodynamics

A.N. Cox, Los Alamos Scientific Laboratory, IAU POB 1663.LOS Alamos, NM 87545

4-9 Aug.

Boulder, CO, USA

Symposium on Atmospheric Ozone

J. London, Dept. of Astro-Geophysics, University of Colorado, Boulder, CO 80309

IAMAP

4-9 Aug.

Ottawa, Canada

7th Int Conf on Raman Spectroscopy

Ken Charbonneau, Conference Services, National Research Council of Canada, Ottawa, Ont., Canada K1A OR6

10-15 Aug.

Helsingớr, Denmark

Int Conf. on Low Dimensional Synthetic Metals K. Carneiro, Physics Laboratory 1, H.C. Ørsted Inst. Universitetsparken 5, DK-2100 Copenhagen $\emptyset$

11-15 Aug.

Radiation in the Atmosphere

Fort Collins, CO, USA

T.H. Vonder Haar, Dept. of Atmospheric Science Colorado State Univ., Fort Collins, CO 80521

Ab: April $80 / P: 11.8 .80 /$ IUGG

11-15 Aug.

Santa Fé, NM, USA

5 th Int. Symposium on Polarization Phenomena in Nuclear Physics

Gerald G. Ohlsen, Los Alamos Scientific Laboratory, Mail Stop 456, Los Alamos, NM 87545

PP / IUPAP / about US \$ 85.-

17-22 Aug

Calgary, Alberta, Canada

Meeting of American Crystallographic Association

K.A. Kerr, Dept. of Chemistry and Physics, University of Calgary, Calgary, Alberta T2N 1N4

17-22 Aug. Santa Cruz, CA USA

5th IUPAC Conf. on Physical Organic Chemistry J.F. Bunnett, Division of Natural Sciences - II, IUPAC University of California, Santa Cruz, CA 95064

\section{7-23 Aug.}

Wolfeboro, NH, USA

Gordon Research Conf.: Vibrational Spectroscopy

E. Ippen, Bell Laboratories, Holmdel, NJ 07733

17-23 Aug.

Eugene, OR, USA

9 th Int. Conf. on the Few-Body Problem

M.J. Moravcsik, Inst. of Theoretical Sciences,

University of Oregon, Eugene, OR 97403

17-23 Aug.

Edmonton, Alberta, Canada

14th Int. Conf. on Thermodynamics and Statistica Mechanics - Stat-Phys 14

D.D. Betts, Stat-Phys 14, Dept. of Physics, University of Alberta, Edmonton, Alberta, Canada T6G 2J1

18-22 Aug.

Brighton, UK

Micro 80

The Administrator, Royal Microscopical Society,

37/38 St. Clements, Oxford OX4 1 AJ

18-22 Aug.

Leeds, UK

int. Conf, on the Physics of Transition Metals

The Meetings Officer, The Institute of Physics

47 Belgrave Square, London SW 1 X $80 X$

24-30 Aug.

Int. Conf. on Nuclear Physics

Berkeley, CA, USA

Berkeley Lab., Berkeley, CA 94720

24-30 Aug.

Prague, Czechoslovakia

it. Conf, on Post-Graduate Education of Physicists

Z. Pluhar, ICPEP Secretariat, Ke Karlovu 3.

CS-12116 Prague 2

A: 31.12.79 / PP / 150 /IUPAP / DM 275.- approx

25-29 Aug.

Fribourg, Switzerland

Int Conf, on Physics in One Dimension

Jernasconi, Brown Boveri Research Centre, CH-5405' Baden

A : 31.3.80/PP: 31.5.80/100/inv. /

Sfr. 100.-, Sfr. 70.- (students)

25-29 Aug.

Strathclyde, UK

$X-R a y$ Processes and Inner-Shell Ionization

D.J. Fabian, Dept. of Metallurgy, Colville Bldg., Univ. of Strathclyde,

48 North Portland Str., Glasgow G1 1XN

A: 1.2 .80 / PP: $31.3 .80 / 400 /$ IUPAP / f 60 .

25-29 Aug.

Melbourne, Australia

4 th Nat. Congress of Australian Inst. of Physics

R.J. Fleming, Dept. of Physics, Monash University Clayton, Victoria 3168

25-30 Aug.

Delft, NL

Joint ISMAR - AMPERE Int. Conf. on Magnetic Resonance

ISMAR-AMPERE 1980, c/o K.I.v.I., 23 Prinsessegracht, РОВ 30424,

The Hague

A : $1.5 .79 /$ PP / 800

25-30 Aug.

Graz, Austria

th Int Microchemical Symposium: Nature, Aim and Methods of Microchemistry

A. Holasek, Institut f. Medizinische Biochemie,

Universität Graz, Harrachgasse 21.

A-8010 Graz

26-29 Aug.

Munich, FRG

10th URSI Symposium on Electromagnetic Theory Coers, VDE Haus, Stresemannallee 21. D-600 Frankfurt 70

28-29 Aug.

Tokyo, Japan

4th Int Conf on Ternary and Multinary Compounds K. Masumoto, Metal Phys. Div., Nat. Res. Inst. for Metals, 2-3-12 Nakameguro, Meguroku, Tokyo 153

Ab: 10.4 .80

Aug. / Sept

Bonn, FRG

Symposium No. 95 : Pulsars

Wielebinski, Max-Planck-Institut für Radioastron

September

Trieste, Italy

Mass Loss from Stars, Physical Mechanisms,

Evolutionary Implications and Interaction with the Interstellar Medium

R. Stalio, Osservatorio Astronomico, I-34131 Trieste 
1-3 Sept.

Antwerp, Belgium

6 th Int. Conf. on High Voltage Electron Microscopy

Symposium on Physical and Metallurgical Applications and Instrumentation

G. Van Tendeloo, Centrum voor Hoogspanningselektronenmikroskopie, RUCA,

Groenenborgerlaan 171, B-2020 Antwerp

PP / 250 / bFr. 3000.-

1-5 Sept.

Conf. on Education for Physics Teaching

International Centre for Theoretical Physic POB 586, I-34100 Trieste

1-6 Sept.

8 th int Congress on Rheology

Nicolais, Montedison DIRS, Via Nuova delle Brecca 150, Barra, Naples

2-5 Sept.

Pisa, Italy

12th EGAS Conference

A. Gozzini, Istituto di Fisica, Università di Pisa, Piazza Torricelli 2, 1-56100 Pisa

2-6 Sept. 4 Oxford, UK

3rd European Study Conf. on Low Energy Molecular Collisions (MOLEC 3 )

M.S. Child, Theoretical Chemistry Dept., University of Oxford, 1 South Parks Road, Oxford OX1 3TG $100 / \mathrm{f} 120$.

3-6 Sept.

Minneapolis, MN, USA

Joint AAS HEAD-APS Cosmic Physics

Division Meeting: Cosmic Ray Astrophysics and Gamma-Ray Line Astronomy

R. Novick, Dept. of Physics, Columbia University, 538 W. 120th Str., New York, NY 10027

3-12 Sept.

Como, Italy

2nd Joint Grenoble-Varenna Int. Conf. on Heating in Toroidal Plasmas

Elio Sindoni, Istituto di Fisica, 16 Via Celoria, 1-20133 Milan

A : 15.5 .80 / PP: 15.6 .80 / 180 / US S 150.-

3-13 Sept.

Dubrovnik, Yugoslavia

3rd Adriatic Meeting on Particle Physics: Recent Developments in Strong Interaction Physics

I. Andric, Ruder Boskovic Institute, POB 1016 YU-41001 Zagreb

A: $31.5 .80 / P P / 80 / D M 750$.-

7-13 Sept.

Int. Symposium on Macromolecules

Florence, Italy

Via Risorgimento $35,1-56100$ Pisa
. Ciardica

IUPAC

8-10 Sept.

Oxford, UK

Photoelectrochemistr

Mrs. Y.A. Fish, The Chemical Society, Burlington House, London WIV OBN

\section{8-11 Sept.}

Canterbury, UK

Physics of Dielectric Solids

The Meetings Officer, The Institute of Physics, 47 Belgrave Square, London SW $1 \times 80 \times$

8-11 Sept. Edinburgh, UK

6 th Int. Conf. on Gas Discharges and their Applications

IEE Conf. Dept. Savoy Place, London WC2R OBL $A b: 26.11 .79$ / PP: 7.4 .80

8-12 Sept.

Warsaw, Poland

2nd Int. Colloquium on Reference Co-ordinate Systems B. Kolaczek, Space Research Centre, Polish Academy of Sciences, Bartycka 18, PL-00-716 Warsaw

IAU, IUGG, COSPAR

8-12 Sept.

Lausanne, Switzerland

2nd Int. Meeting on the Small Particles and Inorganic Clusters

J.-P. Borel, Laboratoire de Physique

Expérimentale, Ecole Polytechnique

Fédérale, 33 av. de Cour,

CH-1007 Lausanne

A: 31.1 .80 / Ab: $\mathbf{3 1 . 1 . 8 0 ~ / ~ P P ~ / ~} 100$ / Engl., French / Sfr. 200. - incl. proc.

8-12 Sept.

Oxford, UK

7 th Symposium on Microdosimetry

-XII, (a) Loi, B-1049 Brussels, Belgium

P: $31.1 .80 / \mathrm{bFr} .2500$.

8-12 Sept.

Munich, FRG

11 th World Energy Conference

Nationales Komitee der Weltenergiekonferenz für die Bundesrepublik Deutschland, Graf-Recke-

Str. 84, Postfach 1139, D-4000 Düsseldorf 1
9-12 Sept.

Bologna, Italy

Erics Conf, on Computing in High

Energy and Nuclear Physics

F. James, DD Division.

CERN, CH-1211 Geneva 23

Ab: March $80 /$ PP: April $80 / 200$

\section{9-12 Sept.}

Budapest, Hungar

9th Symp. IMEKO Tech. Comm. on Photo-Detectors Mrs. J. Solt, IMEKO, POB 457, H-1371 Budapest A: 31.8.80 / Ab: 31.12.79 / PP: 9.9 .80 / 80 US $\$ 70$.

9-12 Sept.

Bochum, FRG

Int. Symposium on Flow Visualization

W. Merzkirch, Institut für Thermo- und Fluiddynamik Ruhr-Univ., POB 102148, D-4630 Bochum

10-12 Sept.

Optics ' 80

Manchester, UK

The Meetings Officer, The Institute of Physics

47 Belgrave Square, London SW 1 X $80 X$

Ab: $1.3 .80 / P: 9.6 .80$

10-16 Sept.

Moscow, USSR

6th Int Conf, on Crystal Growth

P.P. Fedorov, ICCG-6, Institute of Crystallography USSR Academy of Science, Leninski

Prospect 59, Moscow 117333

Ab: 1.2.80/P: 10.4 .80

14-18 Sept.

Cambridge, UK

Physical Chemistry of Microemulsions

I.D. Robb, Unilever Research, Port Sunlight Lab.

Port Sunlight, Wirral, Merseyside, L62 4XN

$\mathrm{Ab}: 1.3 .80$

15-19 Sept.

Electromagnetic Compatibility

P. Elliott, IERE Conf. Dept., 99 Gower Str. London WC1E 6AZ

15-19 Sept. 4

European Solid State Device Research Conference (ESSDERC 1980)

The Meetings Officer, The Institute of Physics, 47 Belgrave Square, London SW1 X $80 \mathrm{X}$

A : July 80/Ab 1.5.80/PP: 15.9.80/300

16-18 Sept.

York, UK IEE Curopean Conf. on Optical Communication $\mathrm{Ab}: 31.3 .80$

20-23 Sept.

8. Convention

BC Secretariat, IEE, Savoy Place,

London WC2R OBL

$A b: 7.1 .80$

22-26 Sept.

Cannes, France

3rd European

8th Int. Vacuum Congress - IVC8

4th Int. Conference on Solid Surfaces (ICSS4)

Société Francaise du Vide, 19 rue du Renard, F-75004 Paris

Ab : 1.1.80 / PP / 1200 / Engl., French, German

22-26 Sept.

Smolenice, Czechoslovakia Hadron Structure ' 80 - QCD-Theory and Experimen A. Nogova, Institute of Physics, Slov. Acad. Sci. CS-899 30 Bratislava

$A: 31.5 .80 / A b: 22.9 .80 / P P: 22.9 .80 / 80 /$ Engl.. Russian / US $\$ 50$

22-26 Sept.

Palaiseau, France

Environment

C. Troyanowsky, Soc. Chimie Physique, 10, rue Vauquelin, F-75005 Paris

Ab: $31.1 .80 / 400$

22-26 Sept.

Beito, Oslo, Norway

SEAS Anniversary Meeting - Workshops: How to Implement Privacy and Security

C.J.M. Aarts, SEAS H.Q., Toernooiveld, Nijmegen, The Netherlands

A: $1.9 .80 / \mathrm{Ab}: 1.6 .80$ / PP: $1.9 .80 / 250$

hFl. 600.-

23-25 Sept.

1980 European Conf. on Optical Systems and Applications

Royal Netherlands Industries Fair Special Events Dept., РОВ 8500, NL-3503 RM Utrecht

Ab: 15.3.80 / NP / Engl. only / hFI. 200.-, hFI. 50. - (students)

23-26 Sept.

Darmstadt, FRG

Nuclear Physics Methods in Material Research

K. Bethge, Institut für Kernphysik, Johann-WolfgangGoethe-Universität, August-Euler-Str. 6 . D-6 Frankfurt-am-Main 90

$\mathrm{PP} / 250$

25 Sept - 1 Oct.

Lausanne, Switzerland

Int. Symposium: High Energy Physics with Polarized Beams and Polarized Targets

C. Joseph, Institut de Physique Nucléaire,

Faculté des Sciences Physiques,

Université de Lausanne,

Dorigny, $\mathrm{CH}-1015$ Lausanne

A : $30.6 .80 /$ Ab : $31.7 .80 /$ PP / $300 /$ Sfr. 100. (incl. proc.)

28 Sept.-5 Oct.

Varna, Bulgaria

Int Symp. Model Investigation of Photographic Process and New Photoregistering Systems

J. Malinowski, Bulgarian Academy of Sciences. 1000 Sofia

29 Sept. - 2 Oct.

Santa Fé, NM, USA

1980 Applied Superconductivity Conference

William E. Keller, LASL, POB 1663, MS 764. 600

30 Sept, - 2 Oct.

Budapest, Hungary

Speech Symposium: Speech Recognition

Acoustical Commission, Hungarian Academy of Science, POB 132, H-1502 Budapest

30 Sept.-3 Oct.

Kyoto, Japan

3rd Int. Conf. on Ferrites (ICF3)

Mitsuo Sugimoto, Dept. of Electronic Engineering, Saitama University, Urawa City 338

A: $1.8 .80 / \mathrm{Ab}: 1.5 .80 / \mathrm{P}: 1.8 .80$

6-9 Oct.

Kyoto, Japan

7th Int. CODATA Conference

B. Dreyfus, Committee on Data for Science and Technology, 51 bd de Montmorency.

F-75016 Paris

6-9 Oct.

Tokyo, Japan

8 th World Computer Congress (IFIP) (also 14-17.10) Information Processing Soc of Japan, Kikai, ShinkiKaikan 3, 5-8 Shiba Koen, Minato-Ku, Tokyo

6-10 Oct.

Lindau/Harz, FRG

Workshop on Acceleration of Particles by Shock Waves throughout Interplanetary and Interstellar Space

A.K. Richter, MPI f. Aeronomie, D-3411 Katlenburg. PP Lindau 3

6-10 Oct.

Berlin-West

5 th Int Conf, on Small-Angle Scattering

R. Hosemann Fritz-Haber-Institut der MPG Teilinstitut $f$. Strukturforschung, Faradayweg 4-6, D-1000 Berlin 33

7-9 Oct.

Baltimore, MD, USA

Electromagnetic Compatibility Symposium

A. Farrar, IIT Research Institute, ECAC/North Severn, Annapolis, MD 21402

7-9 Oct.

Seattle, WA USA

12th National Technical Conf. SAMPE - Materials 1980

Marge Smith, SAMPE, POB 613, Azusa, CA 91702

Ab: $1.2 .80 / P: 1.6 .80$

9-11 Oct.

Minneapolis, MN, USA

Meeting of Nuclear Physics Division (APS)

The American Physical Society, 335E 45th St. New York, NY 10017

9-15 Oct.

Düsseldorf, FRG

8th INTERKAMA

ostfach 70

D-6000 Frankfurt/Main

13-17 0ct.

Amsterdam, NL

2nd Int. Conference of Scientific Editors

Helena Tombal, Elsevier Scientific Publishing Co. POB 330, NL-1000 AH Amsterdam

14-17 Oct. Melbourne, Australia 8th World Computer Congress (IFIP) (also 6-9.10) 8 th World Computer Congress, GPO Box $880 \mathrm{G}$, Melbourne, Victoria, Australia 3001

New Orleans, LA, USA

National Meeting: Optical Society of America 


\section{November}

Allahabad, India

Meeting of Acoustical Society of India

B.S. Ramakrishna, Indian Institute of Science, Bangalore 560012

\section{0-14 Nov.}

San Diego, CA, USA

Meeting of Plasma Physics Division (APS)

The American Physical Society, 335 E. 45th Street, New York, NY 10017

11-14 Nov.

Dallas, Texas, USA

Magnetism and Magnetic Materials

D.C. Bullock. Texas Instruments Inc., РОВ 5936. MS 145 Dallas, TX 75222

24-26 Nov.

Blacksburg. VA, USA

Meeting of Fluid Dynamics Division (APS)

The American Physical Society, 335 E. 45th Street, New York, NY 10017

1-3 Dec.

Los Angeles, CA, USA

Meeting of Electron and Atomic Physics Div, (APS)

The American Physical Society, 335 E. 45th Street, New York, NY 10017

8-10 Dec.

Washington, DC, USA

Int. Electron Devices Meeting

Technical Activities Board, IEEE, 345 E. 47th Street. New York, NY 10017

8-12 Dec.

Miami Beach, FL, USA

Meeting on Infrared and Near Millimeter Waves

K.J. Button, MIT National Magnet Laboratory,

170 Albany Street, Cambridge, MA 02139

16-17 Dec.

Chromatography, Equilibria and Kinetics

Mrs. Y.A. Fish, The Chemical Society, Burlington House, London WIV OBN

\section{1}

5-7 Jan.

18th Annual Solid State Physics Conference The Meetings Officer, The Institute of Physics. 47 Belgrave Square, London SW $1 \times 80 \times$ A: Dec. 80 / Ab: Oct. 80 / NP / 250

\section{9-22 Jan.}

New York, NY, USA

General Meeting of American Physical Society

The American Physical Society, 335 E. 45th Street, New York, NY 10017

27-29 Jan. London, UK

3rd Int. Conference: Future Energy Concepts IEE Conf. Dept., Savoy Place, London WC2R OBL

$A b: 18.2 .80 / P: 18.7 .80$

18-20 Feb.

New York, NY, USA

Int Solid State Circuits Conference

L. Winner, 301 Almeria Ave., Coral Gables, FL 33134

\section{Spring}

Berlin-West

Annual Meeting: German Society of Rheology

K. Kirschke, Unter den Eichen 87, D-100 Berlin 45

A: March 81 /Ab:Sept. 80/PP: Feb. 81/250/Engl., German / DM 120. - approx

11-13 March Washington, DC, USA

Particle Accelerator Conference

S. Penner. National Bureau of Standards.

B104-Radiation Physics, Washington, DC 20234

\section{6-20 March}

Phoenix, Arizona, USA

General Meeting of American Physical Society American Physical Society. 335 E. 45th Street,
New York, NY 10017

30 March - 3 April Karlsruhe, FRG

7 th Int. Conference on Magnet Technology

E.W. Seibt, Karlsruhe Nuclear Research Centre, Institute of Technical Physics.

Mail Box 3640, D-7500 Karlsruhe

$\mathrm{Ab}: 1.11 .80 / \mathrm{PP}: 30.3 .81 / 300$

April
IAU Colloquium No. UK

April
IAU Colloquium No. UK System

R.D. Davies, Royal Astronomical Society, Burlington House, London WIV ONL

\section{6-8 April}

Particle Physics and Nuclear Structure

The Meetings Officer. The Institute of Physics, 47 Belgrave Square, London SWIX $80 X$

6-10 April Heidelberg, FRG

European Conference on Atomic Physics

G. zu Putlitz, Physikalisches Institut der Universität, Philosophenweg 12, D-6900 Heidelberg 1
Leeds, UK

11 th Europhysics Conf. on Macromolecular Physics: Thermal, Mechanical and Electrical Properties of Oriented Polymers

G.R. Davies, Dept. of Physics, University of Leeds. 150 Leeds, LS2 9JT

13-15 April

Bristol, UK

Very High Resolution Molecular Spectroscopy

Mrs. Y.A. Fish. The Chemical Society, Burlington House, London W1V OBN

PP / 200

\section{7-29 April}

Erlangen, FRG

Insulating Films on Semiconductors (INFOS 81)

M. Schulz, Lehrstuhl f. angew. Physik, Universität Erlangen-Nürnberg,Glückstr.9,D-8520Erlangen

27-30 April

Washington, DC, USA

General Meeting of American Physical Society

American Physical Society. 335 E. 45th Street New York, NY 10017

11-15 May

Grenoble, France

International Magnetics Conference (INTERMAG) D. Randet, CEN, B.P. No. 85, Centre de Tri, F-38041 Grenoble Cedex

18-20 May

Santa Fé, NM, USA

International Conference on Plasma Science

S.J. Gitomer, Los Alamos Scientific Laboratory Los Alamos, NM 87545

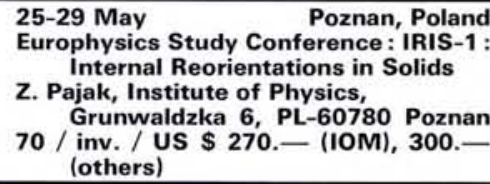

June

Prague, Czechoslovakia

20th Acoustical Conference on Ultrasound

\section{5-17 June}

Los Angeles, CA, USA

Microwave Symposium

A. Clavin, Hugues Aircraft Company, Bldg, 268 , 8433 Fallbrook Ave., Canoga Park, CA 91304

5-10 July

Jerusalem, Israel

4 th Int. Conf. on Surface and Colloid Science

A. Silberberg, Dept. of Polymer Chemistry, Weizmann IUPAC Institute of Science, Rehovo

\section{2-16 July}

Exeter, UK

5 th International NMR Meeting

Shaw, Oxford Instruments Ltd., Osney Mead, Oxford OX2 ODX

\section{3-7 Aug.}

Albuquerque, NM, USA

AU Symposium No. 97 : Extragalactic Radio Sources

.I. Kellerman, NRAO, POB 3 ,

Green

10-14 Aug.

Cryogenic Engineering Conference

San Diego, USA

Int. Cryogenic Materials Conference

Dee Belsher, National Bureau of Standards, Boulder, CO 80303

PP / 500

11-21 Aug. 4

th Int. Conf, on Mathematical Physics - Congress of Association for Mathematical Physics

R. Seiler, Institut f. theor. Physik, FU Berlin, Arnimallee 3, D-1 Berlin 33

16-22 Aug.

Vancouver, Canada 28th Congress: International Union of Pure and Applied Chemistry

UPAC Secretariat, Bank Court Chambers, 2-3 Pound Way, Cowley Centre, Oxford OX4 3YF

19-26 Aug.

Los Angeles, CA, USA

16th Low Temperature Physics Conference (LT 16

R. Orbach. Physics Dept., University of California Los Angeles, CA 90024

\section{3-29 Aug.}

Mexico City, Mexico

7 th Int. Biophysics Congress of International Union for Pure and Applied Biophysics

S. Estrada-O., Director de Ciencias Biologicas y de Salud, Universidad Autonoma Metropolitana Mexico City IUPAB

1-5 Sept.

Tokyo, Japan

22nd Colloquium Spectroscopicum Internationale 9th Int. Conf. on Atomic Spectroscopy

Japan Society for Analytical Chemistry, Gotanda Sanhaitsu, 26-2 Nishigotanda, 1-chome Shinagawa-ku, Tokyo 141

6-12 Sept.

Balaton, Hungary

GIREP Conf. on Physics Education: Nuclear Physics and Nuclear Power

G. Marx, ELTE, Dept. of Atomic Physics, Puskin u 5-7 $\mathrm{H}-1088$ Budapest

150 / US \$ 100. - approx

7-10 Sept.

Linz, Austria

4th Int. Conf. on Small Gap Semiconductors

H. Heinrich, Universität Linz Institut für Physik Experimentalphysik II, A-4045 Linz/Auhof

250 / US \$ 70. - approx.

7-11 Sept.

Grenoble, France

4 th Int. Symposium on Neutron-Capture Gamma-Ray Spectroscopy and Related Topics

T, von Egidy, Physik-Department, E 18, Technische Universität München, James-Franck-Str. D-8046 Garching bei München

$\begin{array}{ll}\text { 7-11 Sept. } & \text { Istanbul, Turkey } \\ \text { 5th EPS General Conference: Trends in Physics }\end{array}$

Istanbul, Turkey

EPS 5, European Physical Society, POB 69, CH-1213 Petit-Lancy 2, Switzerland

14-16 Sept.

Selectivity in Heterogeneous Catalysis Bath BA2 7AY

PP / 200

21-26 Sept.

Leipzig, DDR

12 1 - Eept. Molecular Mobility in Polymer Systems

W. Holzmüller, Dept. of Physics, Karl Marx University. Linnéstr. 5, DDR-701 Leipzig

A: $1.4 .81 / \mathrm{Ab}: 15.3 .81 / \mathrm{NP} / 350 / \mathrm{M} 150$ - (IOM) 180. - (others), approx. 80.- (students)

Late September Moscow, USSR

10th European Conf. on Controlled Fusion and Plasma Physics

October Dubrovnik, Yugoslavia 6th European Regional Astronomy Meeting: Sun and Planetary System

G. Teleki, Astronomical Observatory, Volgina 7. IAU YU-11050 Belgrade

1-7 Oct.

Moscow/Yerevan, USSR

International Symposium on Chemical Physics N.M. Emmanuel, Institute of Chemical Physics. Academy of Sciences of USSR, Vorobyevskoye IUPAC Chaussee 2-6, SU-117334 Moscow

\section{2}

13-17 Sept.

The Neutron and its Applications

Cambridge, UK

P. Schofield, Material Physics Div, B. 521.2 400

AERE, Harwell, Didcot, Oxon, OX11 ORA

\section{Schools 1980}

14-25 Jan. Talence, France

introductory Course in Infrared and Raman Spectroscopy

J.C. Lassegues, Laboratoire de Spectroscopie Infrarouge, 351 Cours de la Libération, F-33405 Talence Cedex

3-14 Feb.

Les Houches, France Ecole d'Hiver: Magnétisme des Métaux et Alliages M. Cyrot, CNRS, 66 X, F-38042 Grenoble Cedex A: 31.10 .79 / PP / 50 / French / FF 4000.-

18-29 Feb. 
18 Feb.-1 March

Karpacz, Poland

Winter School of Theoretical Physics - Recent Developments in the Theory of Fundamental Interactions

L. Rytel, Institute of Theoretical Physics, University of Wroclaw, Cybulskiego 36, Wroclaw

A: $15.11 .79 / 60 /$ US $\$ 170$.

20 Feb.-1 March

Schladming, Austria

19. Int. Universitätswochen für Kernphysik: Field Theory and Strong Interactions

H. Mitter, Org. Comm., Inst. für Theoretische Physik, Universitätsplatz 5, A-8010 Graz

A : $2.2 .80 / \mathrm{PP} / 180$

9-21 March

Erice, Italy

1st Int. School of Climatology: Climatic Variations: Facts and Causes

A. Longhetto, Laboratorio di Cosmo-Geofisica del CRR, Corso Fiume 4, 1-10133 Turin

25 March-6 April Erice, Italy

Int. School of Nuclear Physics: Nuclear Astrophysics

D. H. Wilkinson, University of Sussex, Falmer, Brighton, BN19RH, UK

A: 1.2 .80 / US $\$ 350$.

27 March- 4 April

Erice, Italy

Int. School of Applied Geophysics: Solution of Inverse Problem in Geophysical Interpretation

R. Cassinis, Cattedra di Fisica Terrestre, Università Statale, Via G.B. Viotti 3/5, 1-20133 Milano

A: $15.2 .80 /$ US $\$ 250$.-

8-21 April

Erice, Italy

Int School of Crystallography: New Crystallographic Perspectives in Materials Science

L. Riva di Sanseverino, Istituto di Mineralogia, Piazza San Donato, 1, 1-40127 Bologna

A: 31.10.79/IUCr/Sfr. 600.-

\section{April-20 June}

Trieste, Italy

Spring College on the Physics of Polymers, Liquid Crystals and Low-Dimensional Solids

The Deputy Director, International Centre for Theoretical Physics, POB 586, I-34100 Trieste A: 31.8 .79

\section{5-6 May}

Lillehammer, Norway

Exploration of the Polar Upper Atmosphere

A. Egeland, Institute of Physics, University of Oslo, POB 1038, Blindern, Oslo 3

A: $1.2 .80 / \mathrm{PP} / 65$

21-29 May

Erice, Italy

Advanced School of Geophysics: Geophysics of Geothermal Areas - State of the Art and Future Development

F. Verniani, Istituto di Fisica dell'Atmosfera, Via de Castagnoli, 1-40126 Bologna

A: 1.4 .80 /US $\$ 250$.

26-30 May

Wiezyca, Poland

1 st Spring School on Acousto-optics and its Applications

A. Sliwinski, Institute of Physics, Univ. of Gdansk ul. Wita Stwosza 57, PL-80-952 Gdansk

A: $30.4 .80 / 80 /$ US $\$ 150$.-

1-15 June

Erice, Italy

Int. School of Mathematical Physics: Rigorous Atomic and Molecular Physics

G. Velo, Istituto di Fisica dell'Università

Via Irnerio 46, I-40126 Bologna

8-21 June

Malente, FRG

1980 CERN School of Physics

Miss D.A. Caton, Scientific Conference Secretariat, CERN, CH-1211 Geneva 23, Switzerland

A : $1.3 .80 / 90 /$ Sfr. 800 - all incl.

EPS Divisions, Sections and Group

Astronomy and Astrophysics Division Solar Section

Atomic Physics Division

Atomic Spectroscopy Section

Chemical Physics

Electronic and Atomic Collisions

Molecular Physics

Computational Physics Group

Condensed Matter Division

Low Temperature Sectio

Macromolecular Physics

Magnetism

Metals

Semiconductors and Insulators

Surface and Interface

High Energy \& Particle Physics Division

Nuclear Physics Division

Plasma Physics Division

Quantum Electronics Division
12-20 June

Erice, Italy

Highly Specialized Seminar: Interacting Bose-Ferm System in Nuclei

G. Preparata, CERN, CH-1211 Geneva 23

30 June-11 July

Manchester, UK

7 th UMIST Summer School in Spectroscopic

Interpretation

Registrar, UMIST, POB 88, Manchester M60 IOD

30 June-26 July

\section{aser-Plasma Interactions}

Ecole d'Eté de Physique Théorique,

F-74310 Les Houches

A : 1.3.80 / PP / 50 / FF 2000.- all incl.

1-12 July

Erice, Italy

Int. School of Biophysics: Photoreceptors

A. Borsellino, Istituto di Science Fisiche,

Università di Genova, Viale Benedetto XV, 5 I-16132 Genoa

Erice, Italy
Int. School of Physics of Condensed Matter: Phase

Erice, Italy Transitions and Applications of Ferroelectrics and Related Materials

M. Balkanski, Laboratoire de Physique des Solides, Faculté des Sciences, 9 quai St. Bernard. Paris 5 e, France

2-13 July

St. Croix, Virgin Islands

Advanced Study Institute on Techniques and Concepts of High Energy Physics

T. Ferbel, Fermilab, ASI St. Croix, Mail Station 50 No. 888, POB 500, Batavia, IL 60510

\section{7-31 July} Trieste, Italy

Summer Seminar on Complex Analysis ternational Centre for Theoretical Physics, POB 586, 1-34100 Trieste

A: 30.4 .80

15-24 July Erice, Italy Efficient Use - Information and Analysis

F. Amman, R. Wilson, Istituto di Fisica Applicata dell'Università, Via A. Bassi 6, 1-27100 Pavia

27 July-8 Aug.

Äkäslompolo, Finland

Arctic School of Physics 1980: Current Trends in Particle Physics

Ilkka Liede, University of Helsinki, Dept. of Theor. Phys., Siltavuorenpenger 20

A: $1.3 .80 / 80 /$ Fmk 1900.- (US \$ 500.- approx.)

28 July-8 Aug.

Stanford, CA, USA

SLAC Summer Institute

Anne Mosher, SLAC Summer Institute, POB 4349. Stanford, CA 94305

28 July-30 Aug.

Physics of Defects

Les Houches, France

Ecole d'Eté de Physique Théorique,

F-74310, Les Houches

A : 1.3.80 / PP / 50 / Engl. / FF 2000.- all incl.

31 July-11 Aug.

Int. School of Subnuclear Physics

Erice, Italy

A. Zichichi, CERN, CH-1211 Geneva 23

10-30 Aug.

St. Andrews, UK

Gauge Theories and Experiments at High Energies Alan Walker, Dept. of Physics, University of Edinburgh, Kings Buildings, Mayfield Road, Edinburgh EH9 3JZ

A : 1.5 .80 / PP / 90 / US \$ 350.-

Europhysics News is the official journal of the European Physical Society that comprises $28 \mathrm{Na}$ tional Societies, Academies and Groups, over 3000 Individual Ordinary Members and 30 Associate Members. Governing bodies of EPS are the General Meeting, Council and an elected Executive Committee responsible for detailed policy. EPS promotes the collaboration of physicists throughout Europe and encourages all aspects of international exchange in physics. EPS awards scholarships for research and studies in different countries. EPS publishes, in addition to Europhysics News, Europhysics Conference Abstracts, Euics News, Europhysics Conference Abstracts, Eu-
rophysics Education News and the Proceedings of its General Conferences. Individual Ordinary Members receive Europhysics News (subscription for non-Members: $75 \mathrm{Sw}$. Fr./a), substantial rebates on many publications and pay reduced fees at conferences. Application for membership is made through the permanent Secretariat in Geneva. Annual subscription for members of a National Society from 1 January, 1980 is 32 Sw.Fr.
12-23 Aug.

Dronten, NL

Theol on Nuclear Structure

GAP Engelbertink, Univ. of Utrecht, R.J. van de Graaff Laboratory, Princetonplein 5,

NL 3508 TA Utrecht

A: $1.5 .80 / 100 /$ hFI. 800.- approx.

17-29 Aug.

Erice, Italy

Int. School of Quantum Electronics: Physics and Technology of Free Electron Lasers

A.N. Chester, S. Martellucci, Gruppo Naz. Elettronica Quantistica e Plasmi del CNR, P. le Vincenzo Tecchio, 80, 1-80125 Naples

24 Aug.-28 Sept.

Uppsala, Sweden

International Summer Institute in Quantum Chemistry and Solid State Physics

P.O. Löwdin, Department of Quantum Chemistry, Uppsala University, POB 518

A: 1.5 .80

25 Aug.- 10 Sept. Poiana Brasov, Romania nt. Summer School: Critical Phenomena in Heavy Ion Collisions

Organizing Committee, Central Institute of Physics, Bucharest, Magurele, MG-6

30 Aug.-13 Sept.

Erice, Italy

Int. School of Cosmic Ray Astrophysics: Acceleration of Particles in Nature

M.M. Shapiro, Laboratory for Cosmic Ray Physics Naval Research Laboratory. Washington, DC 20375, USA

31 Aug.-12 Sept.

Mikolajki, Poland Nuclear Structure

Wilhelmi, Hoza 69, PL-00-681, Warsaw

A: $31.5 .80 / \mathrm{PP} / 140 /$ inv. / US \$ 160 .

3-13 Sept.

Erice, Italy

Advanced School of Astronomy: Workshop on Physical Processes in Red Giants

A. Renzini, Osservatorio Astronomico, c.p. 596.

1-40100 Bologna

A: 15.7 .80 / US $\$ 300$.

14-27 Sept.

Vraona-Attiki, Greece

1980 CERN School of Computing

Mrs. I. Barnett, Scientific Conference Secretariat, CERN, CH-1211 Geneva 23

A: $31.3 .80 / 80 /$ approx. Sfr. 800.- all incl.

18 Sept.-1 Oct.

Erice, Italy

int. School of Earth Sciences: Survey of Active Vulcanoes

M. Carapezza, Università degli Studi, Via Archirafi 36 -90123 Palermo

12-22 Nov.

Erice, Italy

int School of Meteorology of the Mediterranean Satellite Technology Applied to the Meteorology of the Mediterranean

A. Nania, Ufficio Ricerche Operative Meteorologia Aeronautica, Aeroporto, 1-72100 Brindisi

\section{1}

July-August

Les Houches, France

Theoretical Physics Summerschool on Chaotic Behaviour in Deterministic Systems

Robert H.G. Helleman, School of Physics, Georgia Tech. Atlanta, GA 30332, USA

Editor : E.N. Shaw

Meetings Compilation: W.S. Newman

Editorial Board :

K. Appert, G.J. Béné, G.R. Macleod,

A. Maeder, J. Muller, D. Pohl

Editorial and Advertising Office at the EPS Secretariat.

Address: EUROPEAN PHYSICAL SOCIETY

P.O. Box 69,

CH-1213 Petit-Lancy 2

Switzerland

Telephone: Geneva (22) 931130

Telex : 23455 alarm ch

Cables: europhys genève

Printed by : Ed. Cherix et Filanosa SA CH-1260 Nyon, Switzerland 